\title{
Series: Cardiovascular outcome trials for diabetes drugs Semaglutide and SUSTAIN-6
}

\author{
MILES FISHER
}

\begin{abstract}
SUSTAIN-6 (Trial to Evaluate Cardiovascular and Other Longterm Outcomes with Semaglutide in Subjects with Type 2 Diabetes) was a pre-licensing FDA-mandated cardiovascular outcome trial with subcutaneous semaglutide and was the first completed trial with a once-weekly glucagon-like peptide-1 receptor agonist. SUSTAIN-6 compared semaglutide and placebo in 3,297 people with type 2 diabetes and established cardiovascular disease, chronic kidney disease, or both, or who were aged over 60 years and had subclinical evidence of cardiovascular disease (persistent microalbuminuria or proteinuria, hypertension with left ventricular hypertrophy, left ventricular dysfunction by imaging, ankle/brachial index less than 0.9). As SUSTAIN-6 was performed as a pre-licensing safety study, non-inferiority for major cardiovascular events (MACE; cardiovascular death, non-fatal myocardial infarction, non-fatal stroke) was the primary outcome and testing for superiority for MACE was not pre-specified or adjusted for multiplicity. SUSTAIN-6 confirmed non-inferiority for MACE, with nominal superiority, and demonstrated a significant reduction in the risk of non-fatal stroke. There was no significant difference in non-fatal myocardial infarction, cardiovascular death, all-cause mortality or hospitalisation for heart failure. Rates of retinopathy complications were unexpectedly higher in the semaglutide group. Further outcome trials with subcutaneous semaglutide are in progress, including a cardiovascular outcome trial in non-diabetic subjects with overweight or obesity.
\end{abstract}

Br J Diabetes 2021;21:110-112

Key words: diabetes, cardiovascular outcome trial, semaglutide

\section{Introduction}

Licensing requirements for new antidiabetic drugs changed in the USA following the rosiglitazone controversy, and between 2008 and 2020 a dedicated randomised controlled cardiovascular out-

Address for correspondence: Professor Miles Fisher Department of Diabetes, Endocrinology \& Clinical Pharmacology, Glasgow Royal Infirmary, 84 Castle Street, Glasgow G4 OSF, UK

E-mail: miles.fisher@ggc.scot.nhs.uk

https://doi.org/10.15277/bjd.2021.301 come trial (CVOT) was usually required either before or after licensing. ${ }^{1}$ This series describes and summarises the results of these CVOTs in the order that they were published, describing the primary endpoint and important secondary outcomes from the principal publication, and directs attention to subsequent publications of data from subgroups and post hoc analyses. SUSTAIN- 6 was the third published FDA-mandated CVOT using a glucagon-like peptide-1 (GLP-1) receptor agonist, and the first with a once-weekly agonist. ${ }^{2}$ It came after one negative trial with the short-acting GLP-1 receptor agonist lixisenatide, ${ }^{3}$ whereas the LEADER trial with liraglutide had demonstrated significant reductions in major adverse cardiovascular events (MACE), cardiovascular deaths and all-cause mortality. ${ }^{4}$

\section{Background}

SUSTAIN-6 was a pre-licensing trial and was completed in 2016. Once-weekly subcutaneous semaglutide was approved in 2017 by the FDA for use in the USA and by the EMA for use in Europe in 2018. Semaglutide was the fourth once-weekly GLP-1 receptor agonist to be approved by the FDA and EMA following approval of once-weekly exenatide, albiglutide and dulaglutide. These three drugs were approved based on cardiovascular safety data from their development programmes, and each was later followed by a dedicated CVOT: exenatide with EXSCEL, ${ }^{5}$ albiglutide with Harmony Outcomes $^{6}$ and dulaglutide with REWIND. ${ }^{7}$

\section{SUSTAIN-6}

The principal results from SUSTAIN-6 were presented in 2016 at the meeting of the European Association for the Study of Diabetes and published simultaneously in the New England Journal of Medicine. ${ }^{2}$ The design of the study and key baseline characteristics of SUSTAIN-6 are described in Box 1.

In SUSTAIN-6 there was non-inferiority for MACE, a composite of cardiovascular death, non-fatal myocardial infarction and non-fatal stroke, with semaglutide (Figure 1, Box 2). Testing for superiority for MACE was not pre-specified or adjusted for multiplicity, but MACE was significantly reduced in the semaglutide group. In the semaglutide group there were statistically significant reductions in an expanded composite outcome (MACE, coronary or peripheral revascularisation, hospitalisation for unstable angina or heart failure) and in non-fatal stroke. There were no significant differences in the rates of non-fatal myocardial infarction, cardiovascular death, death from any cause or hospitalisation for heart failure. Rates of new or worsening 


\section{Box 1 Key features of SUSTAIN-6²}

- SUSTAIN-6 compared semaglutide ( $0.5 \mathrm{mg}$ or $1.0 \mathrm{mg}$ once-weekly) versus placebo for a median follow-up of 2.1 years in 3,297 subjects

- Mean age of subjects was 65 years with a mean duration of diabetes of 14 years

- Mean baseline $\mathrm{HbA}_{1 \mathrm{c}}$ was $8.7 \%$ (72 $\left.\mathrm{mmol} / \mathrm{mol}\right)$

- $60 \%$ of subjects had established ischaemic heart disease, $32 \%$ prior myocardial infarction, $12 \%$ prior ischaemic stroke and $24 \%$ investigator-reported heart failure, but this diagnosis was not well characterised

- $73 \%$ of subjects were on metformin, $43 \%$ sulfonylureas, $3 \%$ meglitinides, $2 \%$ thiazolidinediones, $58 \%$ insulin

Figure 1. 2.1-year event rates (in \%) comparing semaglutide and placebo for major adverse cardiovascular events (MACE), total mortality, cardiovascular mortality, non-fatal myocardial infarction, non-fatal stroke and hospitalisation for heart failure $(\mathrm{HFH})$

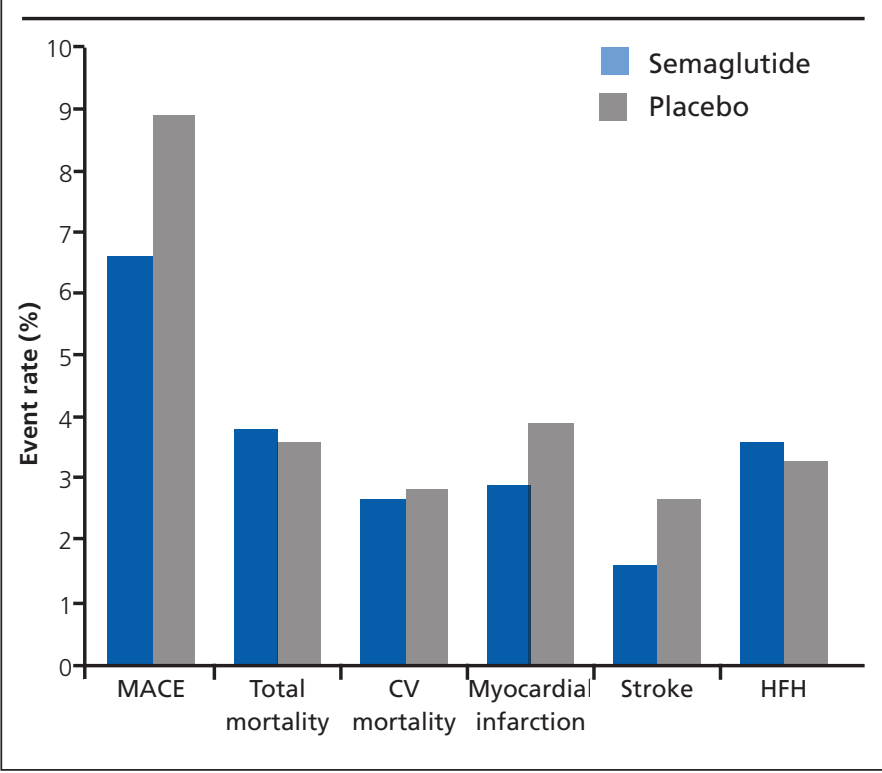

nephropathy were lower in the semaglutide group. The rate of gastrointestinal events was increased with semaglutide and there was an unexpected increase in retinopathy complications with semaglutide (vitreous haemorrhage, blindness or conditions requiring treatment with an intravitreal agent or photocoagulation).

\section{Other results from SUSTAIN-6}

Further publications from SUSTAIN-6 are detailed in Box 2. To get further information about the effects of semaglutide on diabetic retinopathy, data across the SUSTAIN development programme were evaluated and no imbalance in retinopathy was detected. ${ }^{8}$ Further analysis of SUSTAIN-6 showed that the majority of the effects were attributed to the magnitude and rapidity of the reduction in $\mathrm{HbA}_{1 \mathrm{c}}$ during the first 16 weeks of treatment in subjects with pre-existing diabetic retinopathy, poor glycaemia control at baseline, and patients treated with insulin. ${ }^{8}$ The investigators con-

\section{Box 2 Results of the SUSTAIN-6 trial}

Principal result

- $\quad$ Significant reduction in major adverse cardiovascular events (MACE) and non-fatal stroke ${ }^{2}$

\section{Other results from SUSTAIN-6}

- $\quad$ Retinopathy events were increased in the semaglutide group, ${ }^{2}$ and this was associated with large and rapid reductions in $\mathrm{HbA}_{1 \mathrm{c}}$ during the first 16 weeks of treatment, prior diabetic retinopathy, poor glycaemic control and baseline use of insulin ${ }^{8}$

- The risk of MACE was reduced in the semaglutide group regardless of baseline age, gender or cardiovascular risk profile ${ }^{14}$

- Health-related quality of life improved by a greater amount with semaglutide than placebo, possibly mediated in part by changes in body weight and $\mathrm{HbA}_{1 \mathrm{c}}{ }^{15}$

- In post hoc analysis of SUSTAIN-6, nominally significant heterogeneity of semaglutide efficacy by baseline body mass index was observed for MACE, which was not observed in LEADER, and is of uncertain significance ${ }^{16}$

- In SUSTAIN-6 and LEADER, patients with microvascular disease had an increased rate of MACE and semaglutide and liraglutide consistently reduced the risk of MACE in patients with and without microvascular disease ${ }^{17}$

- In SUSTAIN-6 and LEADER, there was an increased frequency of MACE and nephropathy with increasing diabetes duration and semaglutide and liraglutide consistently reduced the risk of cardiorenal outcomes across the categories of diabetes duration ${ }^{18}$

- Combining patient data from SUSTAIN-6 and PIONEER 6 showed consistent effects of semaglutide on MACE across varying degrees of cardiovascular risk, with no effect on MACE in people with prior heart failure ${ }^{19}$

cluded that early worsening of diabetic retinopathy is a previously known phenomenon associated with rapid and large improvements in glycaemic control with insulin, and the findings in SUSTAIN-6 were consistent with this. This 'early worsening phenomenon' related to the speed and degree of improvement in glycaemic control has been accepted by Public Health England. ${ }^{9}$

SUSTAIN- 6 was a pre-licensing CVOT with a smaller number of subjects and lower number of events than most other CVOTs. As an example, the LEADER trial with liraglutide included 9,340 subjects and 1,302 MACE events whereas SUSTAIN-6 included 3,297 subjects with only 254 MACE events. This limits statistical power for further analyses, so there are few other publications from SUSTAIN-6 (Box 2). To get round this problem, further analyses have been performed combining data from SUSTAIN- 6 and LEADER and SUSTAIN-6 and PIONEER 6 (BOx 2).

\section{Discussion}

SUSTAIN- 6 was the first CVOT with a once-weekly GLP-1 receptor agonist and demonstrated significant cardiovascular benefits with a reduction in MACE and non-fatal stroke, and an unexpected increase in diabetic retinopathy. As for the LEADER trial with liraglutide, the separation of cardiovascular events was later than that observed in studies of sodium-glucose co-transporter2 (SGLT2) inhibitors, suggesting a different mechanism of benefit and one that might be related to slowing the progression of atherosclerosis. ${ }^{4}$ There was no imbalance in diabetic retinopathy comparing oral semaglutide and placebo in the pre-licensing PIONEER 6 CVOT. ${ }^{10}$ Further information on the effects of subcu- 


\section{Key messages}

- SUSTAIN-6 was the third published cardiovascular outcome trial with a GLP-1 receptor agonist, comparing semaglutide and placebo

- In SUSTAIN-6, semaglutide significantly reduced major adverse cardiovascular events and non-fatal strokes with no significant effect on cardiovascular death, non-fatal myocardial infarction, all-cause mortality or hospitalisation for heart failure

- Rates of retinopathy complications were significantly higher in the semaglutide group

- Further outcome trials with subcutaneous semaglutide will examine patients with diabetic retinopathy, diabetic kidney disease and non-diabetic subjects with overweight or obesity

taneous semaglutide on the progression of diabetic kidney disease retinopathy will be available from the FLOW renal outcome trial, ${ }^{11}$ and further information effects of subcutaneous semaglutide on the progression of diabetic retinopathy will be available from the FOCUS study. ${ }^{12}$ At present, semaglutide is probably best avoided in patients with significant diabetic retinopathy and liraglutide or dulaglutide are alternatives that have not been associated with worsening of retinopathy. Additional information on the effects of subcutaneous semaglutide on cardiovascular events is being studied in approximately 17,500 overweight and obese subjects in the SELECT trial, with an estimated completion date of September 2023. ${ }^{13}$

Conflict of interest The author has received payment for advisory boards and/or lectures from Allergan, AstraZeneca, Bayer, Boehringer Ingelheim, Daiichi, Eli Lilly, Lexicon, MSD, NAPP, Novo Nordisk and Sanofi.

\section{Funding None.}

\section{References}

1. Chong WH, Yanoff LB, Andraca-Carrera E, Thanh Hai M. Assessing the safety of glucose-lowering drugs - a new focus for the FDA. N Engl J Med 2020;383:1199-202. https://doi.org/10.1056/NEJMp2004889

2. Marso SP, Bain SC, Consoli A, et al, for the SUSTAIN-6 Investigators. Semaglutide and cardiovascular outcomes in patients with type 2 diabetes. N Engl J Med 2016;375:1834-44. https://doi.org/10.1056/NEJMoa1607141

3. Pfeffer MA, Claggett $B$, Diaz R, et al, for the ELIXA Investigators. Lixisenatide in patients with type 2 diabetes and acute coronary syndrome. N Engl J Med 2015;373:2247-57. https://doi.org/10.1056/NEJMoa1509225

4. Marso SP, Daniels GH, Brown-Frandsen K, et al, for the LEADER Steering Committee on behalf of the LEADER Trial Investigators. N Engl J Med 2016;375;311-22. https://doi.org/10.1056/NEJMoa1603827
5. Holman RR, Bethel MA, Mentz RJ, et al, for the EXSCEL Study Group. Effects of once-weekly exenatide on cardiovascular outcomes in type 2 diabetes. N Engl J Med 2017;377:1228-39. https://doi.org/10.1056/NEJMoa1612917

6. Hernandez AF, Green JB, Janmohamed S, et al, for the Harmony Outcomes Committees and Investigators. Albiglutide and cardiovascular outcomes in people with type 2 diabetes and cardiovascular disease (Harmony Outcomes): a double-blind, randomised, placebo-controlled trial. Lancet 2018;392:1519-29. https://doi.org/10.1016/S0140-6736(18)32261-X

7. Gertsein HC, Colhoun HM, Dagenais GR, et al. Dulaglutide and cardiovascular outcomes in type 2 diabetes (REWIND): a double-blind, randomised, placebo-controlled trial. Lancet 2019;394:121-30. https://doi.org/ 10.1016/S0140-6736(19)31149-3

8. Vilsboll T, Bain SC, Leiter LA, et al. Semaglutide, reduction in glycated haemoglobin and the risk of diabetic retinopathy. Diabetes Obes Metab 2018;20:889-97. https://doi.org/10.1111/dom.13172

9. GOV.UK. Diabetic eye screening: cohort management. https://www.gov.uk/government/publications/diabetic-eye-screeningcohort-management-overview/diabetic-eye-screening-cohort-management (accessed 30 April 2021)

10. Husain M, Birkenfeld AL, Donsmark M, et al, for the PIONEER 6 Investigators. Oral semaglutide and cardiovascular outcomes in people with type 2 diabetes. N Engl J Med 2019;381:841-51. https://doi.org/ 10.1056/NEJMoa1901118

11. ClinicalTrials.gov. A research study to see how semaglutide works compared to placebo in people with type 2 diabetes and chronic kidney disease (FLOW). https://clinicaltrials.gov/ct2/show/NCT03819153

12. ClinicalTrials.gov. A research study to look at how semaglutide compared to placebo affects diabetic eye disease in people with type 2 diabetes (FOCUS). https://clinicaltrials.gov/ct2/show/NCT03811561

13. Ryan DH, Lingvay I, Colhoun HM, et al. Semaglutide effects on cardiovascular outcomes in people with overweight or obesity (SELECT) rationale and design. Am Heart J 2020;229:61-9. https://doi.org/10.1016/ j.ahj.2020.07.008

14. Leiter LA, Bain SC, Hramiak I, et al. Cardiovascular risk reduction with once-weekly semaglutide in subjects with type 2 diabetes: a post hoc analysis of gender, age, and baseline CV risk profile in the SUSTAIN 6 trial. Cardiovasc Diabeto/ 2019;18:73. https://doi.org/10.1186/s12933019-0871-8

15. Jódar E, Michelsen M, Polonsky W, et al. Semaglutide improves healthrelated quality of life versus placebo when added to standard of care in patients with type 2 diabetes at high cardiovascular risk (SUSTAIN 6). Diabetes Obes Metab 2020;22:1339-47. https://doi.org/10.1111/ dom.14039

16. Verma S, McGuire DK, Bain SC, et al. Effects of glucagon-like peptide1 receptor agonists liraglutide and semaglutide on cardiovascular and renal outcomes across body mass index categories in type 2 diabetes: results of the LEADER and SUSTAIN 6 trials. Diabetes Obes Metab 2020;22:2487-92. https://doi.org/10.1111/dom.14160

17. Verma S, Bain SC, Honore JB, et al. Impact of microvascular disease on cardiovascular outcomes in type 2 diabetes: results from the LEADER and SUSTAIN 6 clinical trials. Diabetes Obes Metab 2020;22:2193-8. https://doi.org/10.1111/dom.14140

18. Verma S, Bain SC. Fries TM, et al. Duration of diabetes and cardiorenal efficacy of liraglutide and semaglutide: a post hoc analysis of the LEADER and SUSTAIN 6 clinical trials. Diabetes Obes Metab 2019; 21:1745-51. https://doi.org/10.1111/dom.13698

19. Husain M, Bain SC, Jeppesen OK, et al. Semaglutide (SUSTAIN and PIONEER) reduces cardiovascular events in type 2 diabetes across varying cardiovascular risk. Diabetes Obes Metab 2020;22:442-51. https://doi.org/10.1111/dom.13955 